\title{
Associated molecular liquids at the graphene monolayer interface
}

\author{
Florent Goujon, ${ }^{1}$ Aziz Ghoufi, ${ }^{2}$ and Patrice Malfreyt ${ }^{1,}$ a) \\ 1) Université Clermont Auvergne, CNRS, SIGMA Clermont, Institut de Chimie de Clermont-Ferrand (ICCF), \\ F-63000 Clermont-Ferrand, France \\ 2) Université de Rennes, CNRS, IPR (Institut de Physique de Rennes) - UMR 6251, F-35000 Rennes, \\ France
}

(Dated: 2 February 2021)

\begin{abstract}
We report molecular simulations of the interaction between a graphene sheet and different liquids such as water, ethanol and ethylene glycol. We describe the structural arrangements at the graphene interface in terms of density profiles, number of hydrogen bonds (HBs) and local structuration in neighboring layers close to the surface. We establish the formation of a two-dimensional HB network in the layer closest to the graphene. We also calculate the interfacial tension of liquids with a graphene monolayer and its profile along the direction normal to the graphene to rationalize and quantify the strengthening of the intermolecular interactions in the liquid due to the presence of the surface.
\end{abstract}

\section{INTRODUCTION}

Since graphene is used with many organic molecules in electronic organic devices ${ }^{1-5}$, nanopore sequencing ${ }^{6}$, selective filtration ${ }^{7}$, water desalination ${ }^{8,9}$, energy storage $^{10}$, coating materials ${ }^{11}$, strain sensors ${ }^{12}$ and exfoliation processes ${ }^{5}$, the understanding of the properties at the graphene-liquid interface is critical in terms of interactions and local arrangements. The knowledge of the local properties at the interface is an essential step toward the development of graphene-based organic devices. Indeed, the description of the first layers of molecules close to the interface are relevant for many phenomena such as charge transport processes ${ }^{4}$ and adsorption ${ }^{13}$. The structuration of the molecules at the interface is then governed by a balance between specific interactions that can be described through van der Waals, electrostatic or hydrogen bonds energy. These different energy contributions and the organization at the interface should be reflected in a key-property : the solid-liquid interfacial tension.

Indeed, the solid-liquid interfacial tension describes the balance of interactions occurring at the interfacial region. In addition, the knowledge of the liquid-vapor and solidvapor surface tensions then allows the calculation of the contact angle $\theta$ through the Young equation ${ }^{14-16}$. In the case of the interaction between water and graphene, we can address the wettability of graphene (hydrophobic or hydrophilic) through the measurement of the contact angle of a water droplet. However, the measurements of the contact angle are impacted by the nature of the solid surface (roughness and/or chemical heterogeneities, liquid penetration, surface deformation) and the experimental conditions (contamination of the solid substrate by adsorption of hydrocarbons). These experimental difficulties led to scattered values ${ }^{17-21}$ of contact angle ranging from 42 to $125^{\circ}$ giving either a hydrophobic ${ }^{18,22-24}$ or hydrophilic ${ }^{19,20,25}$ property to the graphene.

Theoretical studies also yield controversial conclusions

a)Electronic mail: Patrice.Malfreyt@uca.fr and do not allow to remove the ambiguity on the wetting properties of graphene. Indeed, some quantum calculations ${ }^{26,27}$ have predicted a water contact angle of about $85^{\circ}$ whereas the simulated contact angle with recent atomistic simulations ${ }^{28-31}$ is within the range of 56$100^{\circ}$.

By considering a pristine graphene sheet, it is now possible to calculate directly the graphene-water interfacial tension by molecular simulations ${ }^{31}$ through the stress profile. Indeed, the methodological parameters ${ }^{32-35}$ that have impacted the calculation of the surface tension of liquid-vapor interfaces for a long time (at least 40 years) are now under control and are in the process of being controlled for the calculation of the interfacial tension of solid-liquid interfaces ${ }^{31,36,37}$.

Recently, the $\gamma_{\text {graphene-vapor }}$ interfacial tension was measured experimentally ${ }^{19}$ by using surface force apparatus. It was the first direct measurement of graphene surface energy and $\gamma_{\text {graphene-vapor }}$ found to be equal to $115 \mathrm{mN} \mathrm{m}^{-1}$. Knowledge of this value is a key-element in the use of the Young equation ${ }^{14-16}$ since both interfacial tension $\gamma_{\text {liquid-vapor }}$ and $\gamma_{\text {graphene-liquid }}$ are then accessible by molecular simulation. In the case of the graphene-water interaction, we have established that

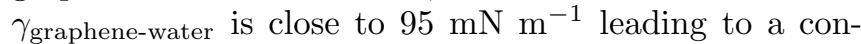
tact angle of approximately $75^{\circ}$ which matches very well with that resulting from surface force apparatus measurements ${ }^{19}$. The graphene surface would be a little more hydrophilic than hydrophobic. In addition, recent atomistic simulations ${ }^{31,38}$ clearly show that water molecules form a double layer of water molecules at the graphene surface. These layers are very wellstructured thanks to a strong local network of hydrogen bonds. This atomistic description establishes that the graphene surface is wettable on an atomic scale ${ }^{38}$. In addition, recent $a b$ initio molecular dynamics (AIMD) simulations ${ }^{39,40}$ have shown that the water molecules are arranged through a two-dimensional (2D) hydrogen bond (HB) network at the liquid-vapor interface ${ }^{39,41}$ and increasing local ordering at the silica-water interface ${ }^{40}$.

Combination of molecular simulations and experimental vibrational sum frequency spectroscopy (VSFS) has 
proved to be relevant for the investigation of the behavior of water molecules and ions ${ }^{42-44}$ at the liquid-vapor ${ }^{45,46}$ and solid-liquid ${ }^{47,48}$ interfaces. The water molecules at the interfacial region show stronger hydrogen bonds ${ }^{49,50}$ and different orientations with respect to bulk conditions. Additionally, molecules that interact with the solid in the first adsorption layer can be subjected to tangential pressures of the order of hundreds of $\mathrm{MPa}^{31,37}$ whereas negative tangential pressure of ten $\mathrm{MPa}$ occur in the liquidvapor interfaces for the same liquid molecules. These interfacial tensions find their molecular origin in a surface effect leading to a compression in the direction parallel to the surface due to the tangential pressure component.

Based on recent developments in the calculation of interfacial tension and on the ability of the simulations to provide a thorough description of the interfacial region in terms of structures, specific arrangements and orientations, we propose here to investigate the adsorption of different molecules which present various polarities and are expected to provide different contact angles ${ }^{51}$ on the graphene sheet. These molecules (ethylene glycol, ethanol and water) differ by the number and strength of the hydrogen bonds leading to variations of the liquidvapor surface tensions ${ }^{52-56}$ from 22 to $72 \mathrm{mN} \mathrm{m}^{-1}$. We aim at calculating the graphene-liquid interfacial tension of these molecules. By comparison with the liquid-vapor surface tension which we will calculate here, we will discuss the role of local arrangements at the graphene surface in relation with the macroscopic interfacial tensions.

We chose the All-Atom (AA) model in order to study more precisely the structure at the interface in terms of hydrogen bonds (donor/acceptor). We are aware that this type of model can lead to surface tensions with larger deviations from experiments compared to United Atoms (UA) models that have reproduced very well this interfacial property ${ }^{57,58}$. Other alternatives are possible and atomistic models that include the polarization by using classical Drude oscillator model ${ }^{59,60}$ have shown to impact significantly the calculation of the work of adhesion of water on graphite and also the contact angle indirectly ${ }^{29}$. However, the use of such models has shown their limitations especially in the reproduction of the liquid-vapor surface tension of salt solutions ${ }^{61}$. On solid-liquid interfaces, the models have been much less tested. The aim of this paper is not to establish the best force field that reproduces the experimental liquidvapor and graphene-liquid interfacial tensions but rather to report the simulated interfacial tensions with the mod- els used here and to rationalize the differences between the graphene-liquid and liquid-vapor interfacial tensions in terms of changes of structuration and local hydrogen bond network. The use of polarizable models is outside the scope of this study.

The paper is organized as follows. Section II contains the description of the model and simulation box as well as the presentation of the calculation of the graphene-liquid interfacial tension. Section III presents the discussion of our results and our conclusions are given in Section IV.

\section{SIMULATION METHODOLOGY}

\section{A. Model}

The total configurational energy $U$ is defined by

$$
U=U_{\mathrm{INTRA}}+U_{\mathrm{INTER}}
$$

where $U_{\text {INTRA }}, U_{\text {INTER }}$ are the intramolecular and intermolecular energy contributions, respectively. The intermolecular contributions sum the repulsion-dispersion and electrostatic energies.

The intramolecular interactions including contributions from the stretching energy, bending energy, torsion energy, and nonbonded dispersion-repulsion and electrostatic interactions are modeled with the General AMBER Force Field (GAFF2) ${ }^{62}$ ) with constrained C-H and O-H bonds.

The intermolecular energy of the system is then

$$
U_{\mathrm{INTER}}=U_{L J}+U_{\mathrm{ELEC}}
$$

where the dispersion-repulsion energy is represented by the truncated Lennard-Jones (LJ) potential of

$$
U_{\mathrm{LJ}}=\sum_{i=1}^{N-1} \sum_{j>i}^{N} \sum_{a=1}^{N_{i}} \sum_{b=1}^{N_{j}} 4 \epsilon_{a b}\left[\left(\frac{\sigma_{a b}}{r_{i a j b}}\right)^{12}-\left(\frac{\sigma_{a b}}{r_{i a j b}}\right)^{6}\right](3)
$$

where $r_{i a j b}$ is the distance between atom $a$ in molecule $i$ and atom $b$ in molecule $j, \epsilon_{a b}$ is the energy parameter of the interaction and $\sigma_{a b}$ is the Lennard-Jones core diameter. $N_{i}$ is the number of atoms in the molecule $i$.

The total electrostatic potential was calculated using the Damped Reaction Field (RFD) version ${ }^{63}$. For a box with orthogonal axes, the electrostatic energy calculated with the RFD formalism is written as 


$$
\begin{aligned}
U_{\mathrm{ELEC}}=U_{\mathrm{RFD}}=\frac{1}{8 \pi \epsilon_{o}} \sum_{i} \sum_{a} \sum_{j \neq i} \sum_{b \neq a} q_{i a} q_{j b} & {\left[\left(\frac{\operatorname{erfc}\left(\alpha r_{i a j b}\right)}{r_{i a j b}}+\left(\frac{\operatorname{erfc}\left(\alpha r_{c}\right)}{r_{c}^{2}}+\frac{2 \alpha}{\sqrt{\pi}} \frac{\exp \left(-\alpha^{2} r_{c}^{2}\right)}{r_{c}}\right) r_{i a j b}\right)\right.} \\
& -\left(\frac{\operatorname{erfc}\left(\alpha r_{c}\right)}{r_{c}}+\left(\frac{\operatorname{erfc}\left(\alpha r_{c}\right)}{r_{c}^{2}}+\frac{2 \alpha}{\sqrt{\pi}} \frac{\exp \left(-\alpha^{2} r_{c}^{2}\right)}{r_{c}}\right) r_{c}\right) \\
& \left.+\frac{B_{0}\left(r_{i a j b}^{2}-r_{c}^{2}\right)}{2 r_{c}^{3}}\right]
\end{aligned}
$$

where $B_{0}$ is defined as

$$
B_{0}=\frac{2\left(\epsilon_{1}-1\right)}{2\left(2 \epsilon_{1}+1\right)}
$$

$\epsilon_{1}$ is the dielectric constant outside the cavity. For water ${ }^{64}$, we take $\epsilon_{1}=78.5$. $\alpha$ was taken to be 0.2365 $\AA^{-1}$. We have shown in a previous work ${ }^{31}$ that the RFD methods compares very well with the Ewald summation technique on the calculation of the graphene-water interfacial tension and the structure of water at the interface.

The LJ parameters for the graphene atom ${ }^{65}$ are $\sigma=$ $3.3997 \AA$ and $\epsilon=0.3594 \mathrm{~kJ} \mathrm{~mol}^{-1}$. The potential parameters including values of $\sigma, \epsilon$ for the water model can be found in the original paper ${ }^{66}$. The LJ parameters for the interactions between unlike sites are calculated by using the Lorentz-Berthelot combining rules. The partial charges of the molecules have been calculated from the density-functional theory (DFT) ${ }^{67,68}(\mathrm{~B} 3 \mathrm{LYP})^{69-71}$ using the Gaussian $16^{72}$ package and the $\mathrm{CHELPG}^{73}$ procedure as a grid-based method. Figure 1a shows the final symmetrized charges of each atom obtained in this work.

\section{B. Definition of the system}

The graphene-liquid interface is modeled by placing the graphene surface as a two dimensional planar material made up of a monolayer of carbon atoms at the center of the box $(z=0)$. The carbon atoms are placed in a hexagonal honeycomb lattice. The system is formed by a graphene sheet surrounded by two reservoirs of molecules. The graphene sheet was kept rigid with no intramolecular interactions between the graphene atoms. The distance $C-C$ was fixed to $1.418 \AA$. The graphene sheet is formed by 2508 carbon atoms leading to a surface area $A=L_{x} L_{y}$ where $L_{x}=81.05 \AA$ and $L_{y}=80.83$ $\AA$.

A liquid-vapor interface has also been simulated for the same three compounds. A bulk liquid phase is first equilibrated in the constant-NVT ensemble; the box size is then increased in the $z$ direction normal to the interface to create a gas phase. Table I summarizes the number of molecules and the box size for the graphene-liquid (SL) interface and the liquid-vapor (LV) interface.
TABLE I. Summary of the box sizes and molecule numbers for the solid-liquid (SL) and liquid-vapor (LV) system simulated in this work.

\begin{tabular}{lcccc}
\hline System & $N_{\mathrm{mol}}$ & $L_{x}$ & $L_{y}$ & $L_{z}$ \\
\hline SL water & 21400 & 81.05 & 80.83 & 101.5 \\
SL ethanol & 6600 & 81.05 & 80.83 & 106.0 \\
SL ethylene glycol & 7000 & 81.05 & 80.83 & 106.0 \\
\hline LV water & 1807 & 30.1 & 30.1 & 250 \\
LV ethanol & 4400 & 60.0 & 60.0 & 300.0 \\
LV ethylene glycol & 4500 & 60.0 & 60.0 & 300.0 \\
\hline
\end{tabular}

The $L_{z}$ dimension of the SL systems was selected from a previous study ${ }^{31}$ in order to avoid any dependence of the interfacial tension on the surface area. Figures $1 \mathrm{~b}$ and 1c show two typical configurations for the SL ethylene glycol and the LV ethanol systems, respectively.
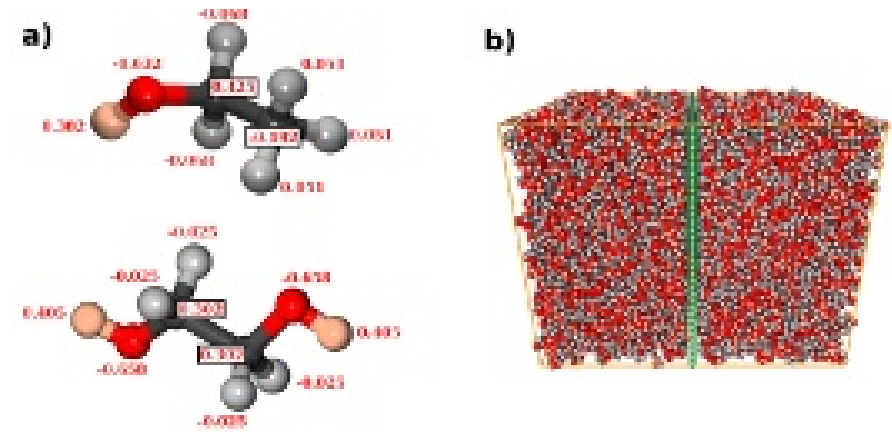

c)

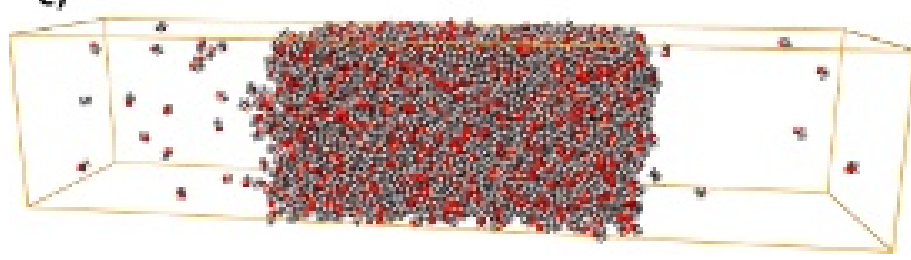

FIG. 1. a) Electrostatic charges calculated on the ethanol and ethylene glycol atoms. b) Typical configuration of a graphene layer in interaction with two reservoirs of ethylene glycol molecules. c) Typical configuration of a liquid-vapor interface of ethanol.

Molecular dynamics (MD) simulations were carried out with the DL_POLY package ${ }^{74}$ by using the Velocity- 
Verlet scheme ${ }^{75}$ to integrate the equations of motion. Periodic boundary conditions were applied along the three directions of space. The SL simulations were carried out in the constant- $\mathrm{N} p_{\mathrm{N}} \mathrm{AT}$ statistical ensemble where the normal component of the pressure $p_{\mathrm{N}}=p_{z z}$ was fixed to 1 bar. The area of the graphene surface is represented by $A$. The temperature $T$ was fixed to $300 \mathrm{~K}$. We applied the Hoover ${ }^{76}$ thermostat and barostat with relaxation times of $\tau_{t}=0.5 \mathrm{ps}$ and $\tau_{p}=0.1 \mathrm{ps}$. The LV simulations were carried out in the constant-NVT ensemble using the Hoover thermostat at $T=300 \mathrm{~K}$ with a relaxation time $\tau_{t}=0.5 \mathrm{ps}$. A time step of $2 \mathrm{fs}$ is used throughout the simulations. The systems were first equilibrated over a period of $5 \mathrm{~ns}$. The thermodynamic and structural properties were then averaged over an acquisition phase of 10 ns using 20000 stored configurations. The LennardJones potential and the electrostatic interactions were truncated at $r_{c}=12 \AA$. Statistical fluctuations of interfacial tensions were estimated using the variation in the averages of five blocks of 4000 configurations.

\section{Graphene-liquid interfacial tension}

The graphene-liquid interfacial tension is calculated by using the mechanical definition of the interfacial tension. We use a local version which is the method of Irving and Kirkwood $(\mathrm{IK})^{77}$. Its expression is

$$
\gamma_{\mathrm{IK}}=\frac{1}{2} \int_{-L_{z} / 2}^{L_{z} / 2}\left(p_{\mathrm{N}}(z)-p_{\mathrm{T}}(z)\right) d z
$$

where $z$ indicates the direction normal to the surface and $p_{\mathrm{N}}(z)$ and $p_{\mathrm{T}}(z)$ are the normal and tangential components of the pressure tensor along this direction, respectively. The factor $1 / 2$ is introduced to consider the two graphene-liquid interfaces of the system.

The method of Irving and Kirkwood ${ }^{77}$ (IK) is based upon the notion of the force across a unit area. The components of the pressure ${ }^{78-80}$ tensor in the Irving and Kirkwood definition are expressed as

$$
\begin{aligned}
p_{\alpha \beta}(z) & =\langle\rho(z)\rangle k_{B} T \mathbf{I} \\
& +\frac{1}{A}\left\langle\sum_{i=1}^{N-1} \sum_{j>i}^{N}\left(\mathbf{r}_{\mathbf{i j}}\right)_{\alpha}\left(\mathbf{F}_{\mathbf{i j}}\right)_{\beta}\right. \\
& \left.\times \frac{1}{\left|z_{i j}\right|} \theta\left(\frac{z-z_{i}}{z_{i j}}\right) \theta\left(\frac{z_{j}-z}{z_{i j}}\right)\right\rangle
\end{aligned}
$$

where $\mathbf{I}$ is the unit tensor and $T$ is the input temperature. $\alpha$ and $\beta$ represent $x, y$ or $z$ directions. $\theta(x)$ is the unit step function defined by $\theta(x)=0$ when $x<0$ and $\theta(x)=1$ when $x \geq 0$. $A$ is the surface area normal to the $z$ axis. The simulation box is divided into $N_{z}$ slabs of thickness $\delta z=0.1 \AA$. Following Irving and Kirkwood, atoms $i$ and $j$ provide a contribution to the pressure tensor in a given slab if the line joining their centres crosses, starts or finishes in the slab. The contribution to a particular slab is $1 /\left|z_{i j}\right|$ of the $i j$ interaction. The normal component $p_{\mathrm{N}}(z)$ is equal to $p_{z z}(z)$ whereas the tangential component is given by $\frac{1}{2}\left(p_{x x}(z)+p_{y y}(z)\right) . \mathbf{F}_{i j}$ in Eq.(7) is the intermolecular force between molecules $i$ and $j$ and is expressed as the sum of all the site-site forces acting between these two molecules.

The long-range corrections ${ }^{81}$ to the surface tensions $\gamma_{\text {LRC }}$ are calculated using Eq.(8) by summing up the local contributions $\gamma_{\text {LRC }}(z)$ of each bin $k$ and dividing the result by a factor 2 .

$$
\begin{aligned}
\gamma_{\mathrm{LRC}}(z) & =\frac{V_{s}}{A}\left(p_{\mathrm{N}, \operatorname{lrc}}(z)-p_{\mathrm{T}, \operatorname{lrc}}(z)\right) \\
& =\frac{\pi}{2} \rho(z) \frac{V_{s}}{A} \int_{r_{c}}^{\infty} d r \int_{-r}^{r} d \Delta z \frac{d u_{\mathrm{LJ}}(r)}{d r} \\
& \times \sum_{i=1}^{N_{s}}\left[\rho\left(z_{i}\right)-\rho\left(z_{i-1}\right)\right]\left[r^{2}-3(\Delta z)^{2}\right]
\end{aligned}
$$

In the case of the graphene-water interfaces, these longrange corrections were found to be negligible ${ }^{31}$. For the liquid-vapor interfaces, the values of these tail corrections are given in Table II.

\section{RESULTS AND DISCUSSIONS}

\section{A. Structure and orientation}

The molecular density profiles along the direction $z$ normal to the graphene sheet is given by

$$
\rho(z)=\frac{1}{A \Delta z}\left\langle\sum_{i=1}^{N} m_{i} H\left(z_{i}-z, \Delta z\right)\right\rangle
$$

where $m_{i}$ is and $z_{i}$ are the mass and $z$-position of the center-of-mass of molecule $i$, respectively. $H$ is the tophat function defined as

$$
H\left(z_{i}-z, \Delta z\right)= \begin{cases}1 & \text { if } z-\frac{\Delta z}{2}<z_{i}<z+\frac{\Delta z}{2} \\ 0 & \text { otherwise }\end{cases}
$$

$A$ is the interfacial area, $\Delta z$ is the slab thickness and $z$ corresponds to the centers of the slabs. $\Delta z$ is set to 0.05 $\AA$ for the SL systems and $0.3 \AA$ for the LV systems.

Figure 2 displays the molecular density profiles of water, ethanol and ethylene glycol in interaction with a graphene sheet up to a distance of $25 \AA$ from the surface in the right part of the simulation box $(z>0)$. We also give for discussion in Figure $2 \mathrm{~b}$ the density profiles of the same species calculated in a planar liquid-vapor interface by centering each one on its Gibbs dividing surface for comparison.

When the molecules interact with a graphene sheet, the density profiles exhibit a number of adsorbed layers and amplitudes that depend on the species studied. The 

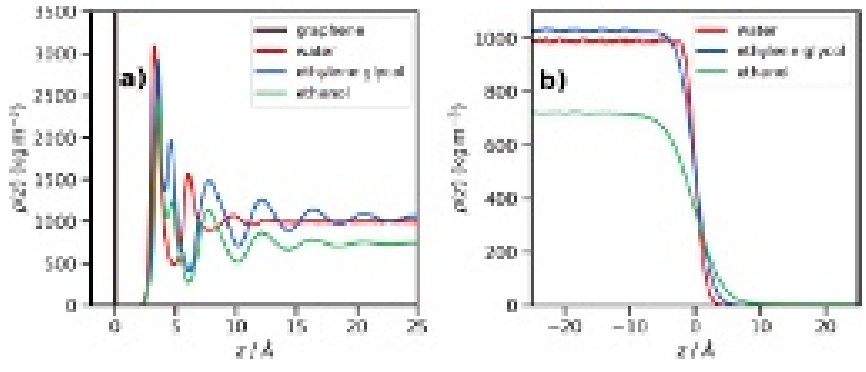

FIG. 2. Molecular density profiles of different molecules as a function of $z$ where $z$ represents the direction normal to the interface in the a) graphene-liquid and b) liquid-vapor interfaces.

width of the oscillations almost corresponds to the size of the molecules. The shape of the first adsorbed layer is also different between water and alcohol molecules. In the case of the liquid-vapor interfaces, the shape of the density profiles are similar between water and ethylene glycol with an interface shape close to a step-function. At the same temperature for ethanol, the interface is wider due to a smaller liquid density and an higher vapor pressure. Figure 2 shows that the molecular density profiles are radically different for species at the interface with graphene and their own vapor.

Figure 3 focuses on the density profiles of adsorbed layers on graphene by extending the region under investigation up to $40 \AA$ from the graphene. We observe in Figure 3 a a first very-well structured adsorption peak of approximately 3 times the value of the bulk liquid density. For water, the first peak is accompanied by a second peak of half the amplitude and a third slight peak located at about $10 \AA$ from graphene. The water then recovers the bulk liquid density at a distance of $12 \AA$ from graphene. The influence of the graphene surface is different with ethanol and ethylene glycol. Indeed, the first peak shows a shoulder whose contributions come from both carbon and oxygen atoms. There is a kind of sub-structure in this first layer for alcohol molecules due to the balance in this well-ordered layer between the strong structuration imposed by the formation of hydrogen bond (HB) and the preference for the methylene groups to interact with the graphene. The investigation of the hydrogen bond network will be proposed later in this paper. For these alcohol molecules, it is very interesting to note that the formation of structured layers extends over $20 \AA$ and 25 $\AA$ for ethanol and ethylene glycol, respectively. This increase in the region of interaction in the $z$-direction is explained in part by the size of the molecule and a stronger correlation between alcohol molecules and graphene.

The layering of molecules in the interface region is also the result of an adaptation of the 3D HB network of bulk conditions to a 2D-geometry imposed by the interaction with a graphene surface. Figure 4 shows the number of hydrogen bonds per donor as function of the $z$-position. This number is normalized by the number of potential
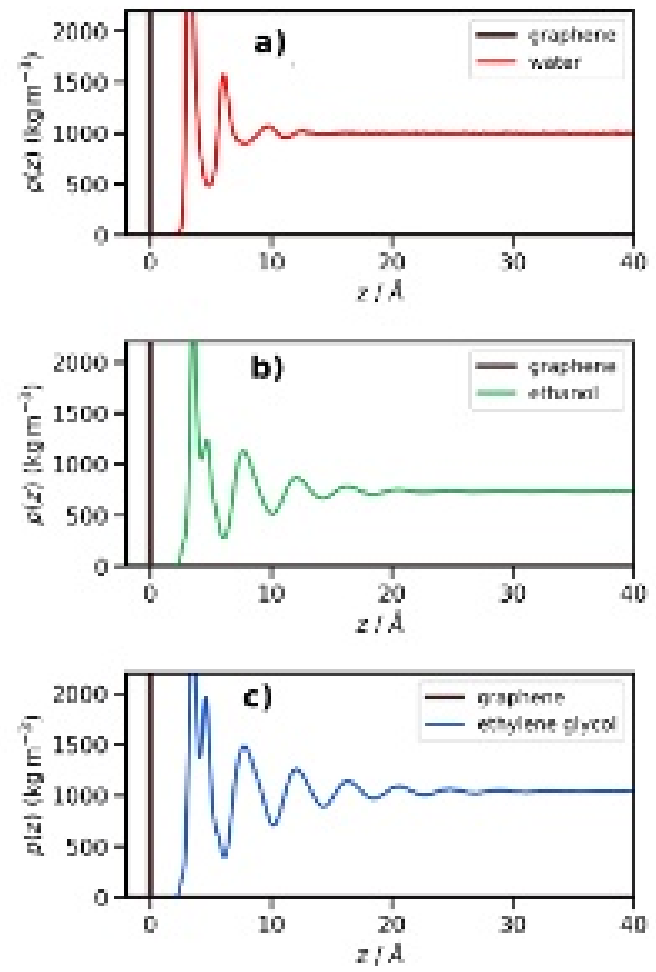

FIG. 3. Molecular density profiles $\rho(z)$ calculated over $40 \AA$ along the $z$-direction for a) water, b) ethanol and c) ethylene glycol molecules. We have limited the scale on the y-axis to $2000 \mathrm{~kg} \mathrm{~m}^{-3}$ to have a better view of successive peaks with decreasing amplitudes.

donors (two for water and ethylene glycol and one for ethanol). The criteria used to select an hydrogen bond have been taken from Ref.82. Two water molecules are chosen as being hydrogen bonded only if the distance (O-H..O) between the donor and acceptor is less than $2.5 \AA$ and simultaneously the angle O-H..O is greater than $135^{\circ}$. As each water molecule can give two HB and receive two $\mathrm{HB}$, we obtain the classical value of 3.7 HB per water molecule in the bulk region by multiplying this number by 4 . Interestingly, the position of the first peak of density (see Figure 3) is also the maximum of hydrogen bonds. These profiles show that the expected number of hydrogen bond by donor in bulk conditions is recovered at about $15 \AA$ to the surface. For water close to the surface, this number is increased by $5 \%$ with respect to the bulk liquid water phase whereas an increase of 12 and $18 \%$ is observed for ethanol and ethylene glycol with respect to bulk conditions.

Figure 5 shows the 2D-distributions of the cosine value of the angle between the $\mathrm{O}-\mathrm{H}$ donor vector and the normal to the surface as a function of the position $z$ from the graphene surface. The first layer corresponds to the first peak of density. For water, we observe a very strong orientation of water molecules in the first layer which im- 


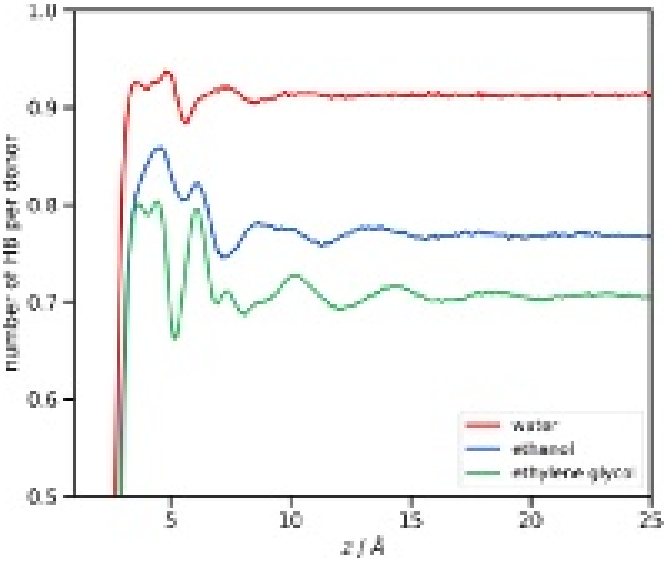

FIG. 4. Number of hydrogen bonds normalized by the number of donors ( 2 for water and ethylene glycol) as function of the distance to the graphene surface for water, ethylene glycol and ethanol.

plies a lesser degree of orientation in the second layer. At a distance greater than $10 \AA$ from the graphene, the distribution of the $\theta$ angle becomes flat indicating random orientations as expected for bulk conditions. These observations are quantified in the distributions of $\cos \theta$ in Figure $6 \mathrm{a}$ for the first layer. The peak of the distribution for water is located at about $\cos \theta=-0.1$ suggesting that a large fraction of molecules orient the $\mathrm{O}-\mathrm{H}$ vector perpendicular to the normal to the graphene. In other words, the water molecules closest to the graphene have an $\mathrm{O}-\mathrm{H}$ bond parallel to the surface. This strong orientation of water molecules in the first layer also impacts the second layer by pushing the water molecules of this layer to take two preferred orientations (see Figures 5 and $6 \mathrm{~b})$ : when the oxygen atom of water molecules of the first layer acts an a acceptor of hydrogen bond, preferred orientations occur in the second layer with $\mathrm{O}-\mathrm{H}$ bond perpendicular to the graphene surface $(\cos \theta=-1)$; when the $\mathrm{O}-\mathrm{H}$ bond of water molecules in the first layer plays the role of donor, water molecules of the second layer are now oriented with one $\mathrm{O}-\mathrm{H}$ bond both pointing toward the liquid phase with $\cos \theta=1$. To conclude, we observe a very strong structuration of the two first layers : a first layer with hydrogen-bonds mainly oriented parallel to the surface and a second layer characterized by a majority of hydrogen bonds perpendicular to the graphene. It means that when water molecules approach the surface, there is a broken symmetry that prevents water molecules to form HBs as they do in the bulk liquid. As a result, they arrange to point the oxygen atoms toward the surface and the $\mathrm{O}-\mathrm{H}$ bonds parallel to the surface forming then a $2 \mathrm{D}$ HB network. The number of hydrogen bonds per donor is then increased with respect to bulk conditions (see Figure 3a). Recently, many-body electronic structure methods ${ }^{30}$ have shown that the orientation of a water monomer on graphene does not impact on the adsorption energy. It means that the specific orientation of water molecules in the first layer close to the graphene monolayer is explained by the fact of maintaining a strong local network of hydrogen bonds in the interfacial region and not by the interaction with the graphene. Figure $6 \mathrm{c}$ shows the distributions of $\cos \theta$ in the region of the Gibbs dividing surface of a liquid-vapor interface. Of course, the distributions are wider with respect to the graphene interface but we can conclude that the water molecules exhibit a certain orientation and structuration in this interfacial region as recently shown by $a b$ initio molecular dynamics calculations ${ }^{41,83,84}$. The sphere of hydration is broken and the molecule reorganizes itself by creating an excess density.
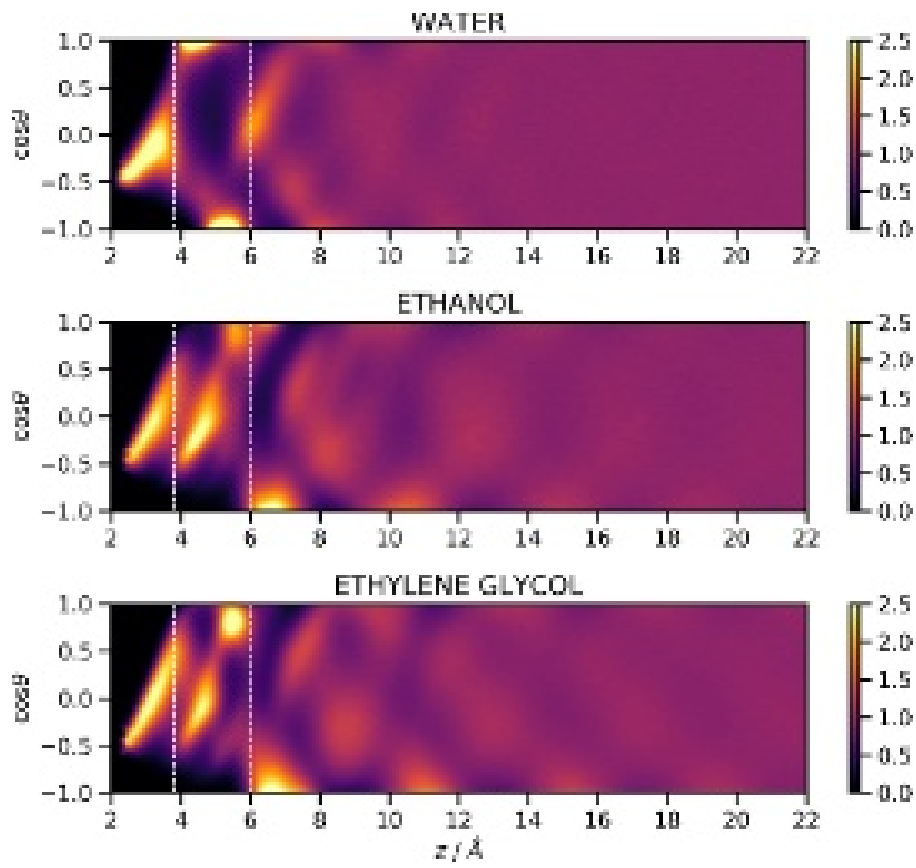

FIG. 5. 2D distribution of the $\mathrm{OH}$ vectors that give a hydrogen bond as a function of their position $z$ for the three systems. Layer 1 is the closest to the graphene surface, located at $z=0$. $\theta$ is the angle between the $\mathrm{OH}$ vector and the $z$ axis normal to the surface. The colors represent the relative probability with respect to the random orientation found in the bulk region, set to unity. The dashed lines show the two first layers.

We now focus on the structuration of alcohol molecules in the graphene layer and liquid-vapor interfaces. The strong orientation observed with water molecules within a 2D organisation of hydrogen bonds parallel to the interface also applies to alcohol molecules as shown Figures 5 and 6 . The main difference with water lies in a flatter angle distribution $n(\cos \theta)$ which is centered on $<\cos \theta>=0$. For alcohol molecules, the $\mathrm{O}-\mathrm{H}$ donor has the possibility to be more parallel to the surface than with water molecules, certainly due to a less constrained network of hydrogen bonds. The second layer also shows slight differences in the distribution of $\theta$ with especially privileged orientations characterized by $\langle\cos \theta\rangle=0$ for 
ethanol and ethylene glycol and another set of favored orientations at $\langle\cos \theta>=0.75$. The 2D-HB network is maintained in the second layer, albeit to a lesser extent. This can be explained by the size of the molecules and the number of consecutive oscillating peaks in the density profiles in Figure 3. As regards the liquid-vapor interface, we also note a slight orientation of alcohol molecules parallel to the interface to a lesser degree than water. Let us recall that even in bulk conditions, alcohol molecules form chainlike structures ${ }^{85-88}$ which are less cohesive than the 3D-HB network of water.
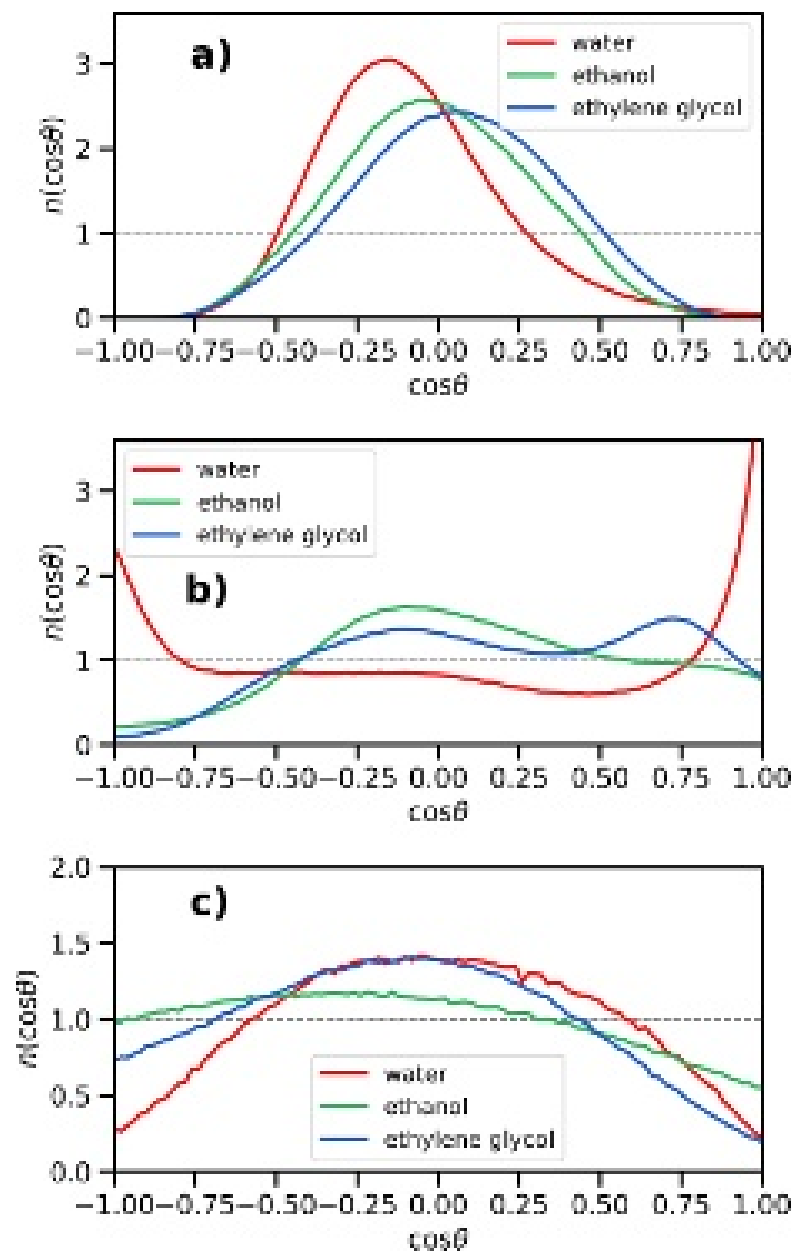

FIG. 6. $n(\cos \theta)$ distributions calculated for water and alcohol molecules for a) the first layer defined by a maximum distance to the graphene of $4 \AA$; b) the second layer defined by $4<$ $z<6 \AA$; c) a region extending over $10 \AA$ from the position of the Gibbs dividing surface in the liquid-vapor interface.

\section{B. Interfacial tensions}

We now turn our attention on the values of interfacial tensions calculated in graphene-liquid and liquid-
TABLE II. Interfacial tensions of the liquid-vapor and graphene-water interfaces. The different contributions of the interfacial $\gamma_{\mathrm{LJ}}, \gamma_{\mathrm{ELEC}}$ and $\gamma_{\mathrm{LRC}}$ are given for discussion. The average interfacial tension $\langle\gamma\rangle$ sums up the three parts. The subscripts give the accuracy of the last decimal(s), i.e., 108.7 $7_{90}$ means $108.7 \pm 9.0$.

\begin{tabular}{lcccc}
\hline System & $\gamma_{\mathrm{LJ}}$ & $\gamma_{\mathrm{ELEC}}$ & $\gamma_{\mathrm{LRC}}$ & $\langle\gamma\rangle$ \\
& \multicolumn{5}{c}{$\left(\mathrm{mN} \mathrm{m}^{-1}\right)$} \\
\hline Water (SL) & -479.2 & 587.9 & 0 & $\mathbf{1 0 8 . 7}_{90}$ \\
Water (LV) & -244.6 & 312.2 & 4.4 & $\mathbf{7 2 . 0}_{105}$ \\
& & & & \\
Ethanol (SL) & -24.8 & 92.0 & 0 & $\mathbf{6 7 . 2}_{18}$ \\
Ethanol (LV) & 1.5 & 13.3 & 3.9 & $\mathbf{1 8 . 7}_{17}$ \\
& & & & \\
Ethylene glycol (SL) & -195.0 & 269.3 & 0 & $\mathbf{7 4 . 3}_{26}$ \\
Ethylene glycol (LV) & -105.7 & 137.1 & 6.3 & $\mathbf{3 7 . 7}_{28}$ \\
\hline
\end{tabular}

vapor interfaces. These interfacial tensions are calculated with the Damped Reaction Field (RFD) method. The good agreement obtained with the experimental and simulated ${ }^{66,89}$ liquid-vapor surface tension of water at $298 \mathrm{~K}$ establishes that the RFD approach performs very well in the calculation of this property with the same accuracy as the Ewald method ${ }^{89}$. In addition, the Lennard-Jones and electrostatic contributions calculated with RFD for this liquid-vapor interface are also in line with those calculated with the Ewald summation method at a higher temperature ${ }^{89}(\mathrm{~T}=478 \mathrm{~K})$. Concerning the graphene-water interfacial tension, RFD reproduces the value calculated with Ewald ${ }^{31}$. For alcohol, we use the General AMBER Force Field (GAFF2) ${ }^{62}$ based on an allatom description that has not been optimized on coexisting properties. This can explain some deviations from experiments on the liquid-vapor surface tensions of alcohol molecules for which United Atom models ${ }^{57,58}$ perform better. The aim of this paper is to rationalize the changes in the interfacial tensions between liquids and a graphene layer and liquid-vapor in line with the structural arrangements at the interface. The different contributions to the interfacial tensions are given in Table II.

From a methodological viewpoint, Figure 7c shows the profiles of the difference between the normal and tangential components of the pressure tensor and the integral $\gamma(z)$ for the water LV system. The profiles show features that are in line with the mechanical equilibrium of planar liquid-vapor interface : two peaks of about $60 \mathrm{MPa}$ at the two interfaces and no contribution from the bulk liquid and vapor phases leading to constant local values of $\gamma(z)$ in these bulk phases.

The analysis is more challenging for the interaction of liquids with a graphene monolayer. Indeed, each density peak is associated with a peak of the $p_{N}(z)-p_{T}(z)$ profile. A drop of $p_{N}(z)-p_{T}(z)$ from 400 to more than -1000 $\mathrm{MPa}$ is calculated in the first layer of water molecules corresponding to a thickness of about $5 \AA$ leading to a local interfacial tension of about $125 \mathrm{mN} \mathrm{m}^{-1}$ in line with a 
strong associated fluid. This profile is radically different to that observed in the liquid-vapor interface establishing that the different consecutive layers of molecules are marked by different local interfacial tensions. The strong orientation and structuration of the molecules due to the presence of the graphene clearly appear through the local values of pressures and interfacial tensions.
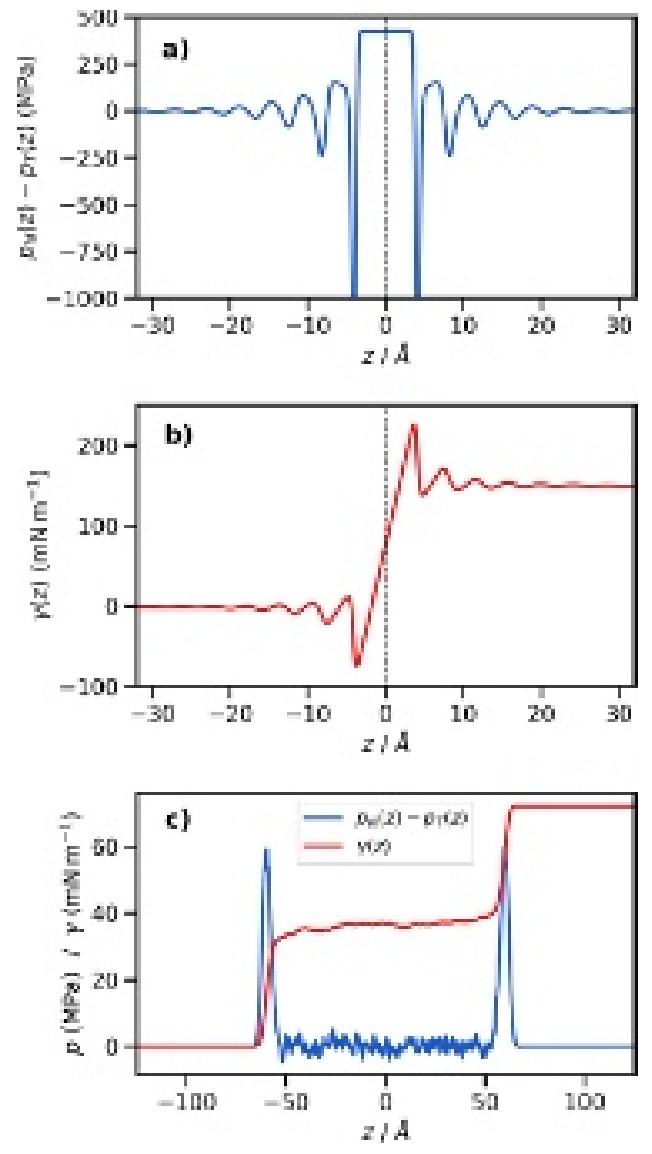

FIG. 7. Difference of $p_{N}(z)-p_{T}(z)$ (MPa) pressure profiles calculated in a) the graphene-water interface and c) the liquidvapor interface of water. The integral $\gamma(z)$ of the local interfacial tension $\left(\mathrm{mN} \mathrm{m}^{-1}\right)$ calculated using Eq.(6) is given in b) for graphene-water and in c) for water liquid-vapor systems.

For completeness, Table II lists the different contributions to the surface tensions. A negative contribution to the surface tension from the Lennard-Jones (LJ) part means that the LJ interacting centers are on average at a smaller distance than the $\sigma$ diameter parameter leading to positive values for the energy. This repulsive LJ energy is then explained by strong electrostatic interactions. The magnitude of the LJ part to the interfacial tension measures to a certain extent the strength of the association in the liquid. For water and ethylene glycol, the LJ contribution to the surface tension becomes much more negative when we move from the liquid-vapor to the graphene-water interfaces. For ethanol, this contribution changes from a positive to a negative value but it is also the lowest value of the systems studied here. From an energetic viewpoint, the strengthening of the association in the fluid due to the graphene is the most significant for water with $\gamma_{\mathrm{SL}}=108.7 \mathrm{mN} \mathrm{m}^{-1}$ and the lightest for ethanol with $\gamma_{\mathrm{SL}}=67.2 \mathrm{mN} \mathrm{m}^{-1}$. We do not see significant changes in the structuration of the two closest layers next to the graphene for the alcohol molecules in line with similar interfacial tensions.

\section{CONCLUSIONS}

The graphene-liquid interface was investigated by means of molecular simulations. The density profiles along the direction normal to the surface establish different adsorbed layers with a number and amplitudes that depend on the molecule. The shape of the profiles changes radically from those calculated in a liquid-vapor interface.

The simulations have evidenced a very strong structuration in the two layers closest to the graphene. As water and alcohol molecules approach the graphene, they undergo a break in symmetry that cancels any possibility of forming hydrogen bond on the side of graphene. Molecules adapt to this situation by orienting the hydrogen bonds in the direction parallel to the surface leading to a $2 \mathrm{D} \mathrm{HB}$ network in the first layer. The interfacial region of the liquid-vapor equilibrium also shows the formation of $2 \mathrm{D} \mathrm{HB}$ network but to a lesser extent compared to graphene-liquid interface.

The layering of water and ethanol molecules when they interact with the graphene is noticeable on the profiles of interfacial tension and pressure components. In the case of water, the first layer of water sees a drop of $p_{N}-p_{T}$ of about $1500 \mathrm{MPa}$ over a thickness of $5 \AA$. In this first layer, the local interfacial tension is increased by $74 \%$ with respect to the surface tension of liquid-vapor interface. From an energetic viewpoint, the enhancement of the interaction within the liquid due to the graphene is the most significant for water and the smallest for ethanol.

This paper aimed at showing how properties of associated liquids characterized by different hydrogen bond strengths change with respect to bulk conditions when they interact with a surface. The structural changes due to the adsorption have been analyzed in terms of local molecular density and orientations. Some dramatic changes in the pressure tensor and local surface tensions have been identified in the interfacial region with the solid. These structural and energetic modifications are significantly different from those observed in the interfacial region of the liquid-vapor equilibrium.

\section{DATA AVAILABILITY}

The data that support the findings of this study are available from the corresponding author upon reasonable request. 
${ }^{1}$ S. Coe, W.-K. Woo, M. Bawendi, and V. Bulovic, "Electroluminescence from single monolayers of nanocrystals in molecular organic devices," Nature 420, 800-803 (2002).

${ }^{2}$ F. Bonaccorso, Z. Sun, T. Hasan, and A. C. Ferrari, "Graphene photonics and optoelectronics," Nat. Photonics 4, 611-622 (2010).

${ }^{3}$ K. Kim, J. Y. Choi, T. Kim, S. H. Cho, and H. J. Chung, "A role for graphene in silicon-based semiconductor devices," Nature 479, 338-344 (2011).

${ }^{4}$ G. Hong, Q. H. Wu, J. Ren, C. Wang, W. Zhang, and S. Lee, "Recent progress in organic molecule/graphene interfaces," Nano. Today 8, 388-402 (2013).

${ }^{5}$ A. Schlierf, P. Samori, and V. Palermo, "Graphene-organic composites for electronics: Optical and electronic interactions in vacuum, liquids and thin solid films," J. Mater. Chem. C 2 (2014).

${ }^{6}$ S. Garaj, W. Hubbard, A. Reina, J. Kong, D. Branton, and J. A. Golovchenko, "Graphene as a subnanometre trans-electrode membrane," Nature 467, 190-193 (2010).

${ }^{7}$ S. C. O'Hern, C. A. Stewart, M. S. H. Boutilier, J. C. Idrobo, S. Bhaviripudi, S. K. Das, J. Kong, T. Laoui, M. Atieh, and R. Karnik, "Selective molecular transport through intrinsic defects in a single layer of cvd graphene," ACS Nano 6, 10130-10138 (2012).

${ }^{8}$ D. Cohen-Tanugi and J. C. Grossman, "Water desalination across nanoporous graphene," Nano Lett. 12, 3602-3608 (2012).

${ }^{9}$ L. Garnier, A. Szymczyk, P. Malfreyt, and A. Ghoufi, "Physics behind water transport through nanoporous boron nitride and graphene," J. Phys. Chem. Lett. 7, 3371-3376 (2016).

${ }^{10}$ Y. Zhu, S. Murali, M. D. Stollernd, K. J. Ganesh, W. Cai, P. J. Ferreira, A. Pirkle, R. M. Wallace, K. A. Cychosz, M. Thomme, D. Suand, E. A. Stach, and R. S. Ruoff, "Carbon-based supercapacitors produced by activation of graphene," Science 332, 15371541 (2011).

${ }^{11}$ M. Topsakal, H. Sahin, and S. Ciraci, "Graphene coatings: An efficient protection from oxidation," Phys. Rev. B 85, 155445 (2012).

${ }^{12}$ M. Oliva-Leyva and C. Wang, "Low-energy theory for strained graphene: an approach up to second-order in the strain tensor," J. Phys.: Condens. Matter 29, 165301 (2017).

${ }^{13}$ G. Ersan, O. G. Apul, F. Perreault, and T. Karanfil, "Adsorption of organic contaminants by graphene nanosheets: A review," Water Res. 126, 385-398 (2017).

${ }^{14} \mathrm{~T}$. Young, "An essay on the cohesion of fluids," Philos. Trans. R. Soc. London 95, 65 (1805).

${ }^{15} \mathrm{~A}$. Marmur, "Soft contact: measurement and interpretation of contact angles," Soft Matter 2, 12-17 (2006)

${ }^{16}$ E. Bormashenko, "Physics of solid-liquid interfaces: From the young equation to the superhydrophobicity," Low Temperature Physics 42, 622-635 (2016).

${ }^{17}$ A. Ashraf, Y. Wu, M. C. Wang, N. R. Aluru, S. A. Dastgheib, and S. Nam, "Spectroscopic investigation of the wettability of multilayer graphene using highly ordered pyrolytic graphite as a model material," Langmuir 30, 12827-12836 (2014).

${ }^{18}$ T. Ondarçuhu, V. Thomas, M. Nunez, E. Dujardin, A. Rahman, C. T. Black, and A. Checco, "Wettability of partially suspended graphene," Sci. Rep. 6, 24237 (2016)

${ }^{19}$ C. D. van Engers, N. E. A. Cousens, V. Babenko, J. Britton, B. Zappone, N. Grobert, and S. Perkin, "Direct measurement of the surface energy of graphene," Nano Lett. 17, 3815-3821 (2017).

20 A. V. Prydatko, L. A. Belyaeva, L. Jiang, L. M. Lima, and G. F. Schneider, "Contact angle measurement of free-standing squaremillimeter single-layer graphene," Nat. Commun. 1 (2018).

${ }^{21} \mathrm{~J}$. Feng and Z. Guo, "Wettability of graphene: from influencing factors and reversible conversions to potential applications," Nanoscale Horiz. 4, 339-364 (2019).

${ }^{22}$ S. Wang, Y. Zhang, N. Abidi, and L. Cabrales, "Wettability and surface free energy of graphene films," Langmuir 25, 11078-11081 (2009).
${ }^{23}$ G.-T. Kim, S.-J. Gim, S.-M. Cho, N. Koratkar, and I.-K. Oh, "Wetting-transparent graphene films for hydrophobic waterharvesting surfaces." Adv. Mater. 26, 5166-5172 (2014).

${ }^{24}$ M. Munz, C. E. Giusca, R. L. Myers-Ward, D. K. Gaskill and O. Kazakova, "Thickness-dependent hydrophobicity of epitaxial graphene thickness-dependent hydrophobicity of epitaxial graphene," ACS Nano 9, 8401-8411 (2015).

${ }^{25}$ A. Kozbial, Z. Li, C. Conaway, R. McGinley, S. Dhingra, V. Vahdat, F. Zhou, B. D'Urso, H. Liu, and L. Li, "Study on the surface energy of graphene by contact angle measurements," Langmuir 30, 8598-8606 (2014).

${ }^{26} \mathrm{H}$. Li and X. C. Zeng, "Wetting and interfacial properties of water nanodroplets in contact with graphene and monolayer boronnitride sheets," ACS Nano 6, 2401-2409 (2012).

${ }^{27} \mathrm{~J}$. Li and F. Wang, "Water graphene contact surface investigated by pairwise potentials from force- matching paw-pbe with dispersion correction," J. Chem. Phys 146, 054702 (2017).

${ }^{28}$ F. Taherian, V. Marcon, N. F. A. van der Vegt, and F. Leroy, "What is the contact angle of water on graphene?" Langmuir $\mathbf{2 9}$ 1457-1465 (2013).

${ }^{29}$ R. P. Misra and D. Blankschtein, "Insights on the role of manybody polarization effects in the wetting of graphitic surfaces by water," J. Phys. Chem. C 121, 28166-28179 (2017).

${ }^{30}$ J. G. Brandenburg, A. Zen, M. Fitzner, B. Ramberge, G. Kresse, heodoros Tsatsoulis, A. Grüneis, A. Michaelides, and D. Alfe, "Physisorption of water on graphene: Subchemical accuracy from many-body electronic structure methods," J. Phys. Chem. Lett. 10, 358-368 (2019).

${ }^{31}$ T. Dreher, C. Lemarchand, N. Pineau, E. Bourasseau, A. Ghoufi, and P. Malfreyt, "Calculation of the interfacial tension of the graphene-water interaction by molecular simulations," J. Chem Phys. 150, 014703 (2019).

${ }^{32}$ F. Goujon, P. Malfreyt, A. Boutin, and A. H. Fuchs, "Vapourliquid phase equilibria of n-alkanes by direct monte carlo simulations," Mol. Simul. 27, 99-114 (2001).

${ }^{33}$ A. Ghoufi, F. Goujon, V. Lachet, and P. Malfreyt, "Multiple histogram reweighting method for the surface tension calculation," J. Chem. Phys. 128, 154718 (2008).

${ }^{34}$ X. Paredes, J. Fernandez, A. A. H. Padua, P. Malfreyt, F. Malberg, B. Kirchner, and A. S. Pensado, "Bulk and liquid-vapor interface of pyrrolidinium-based ionic liquids: A molecular simulation study," J. Phys. Chem. B 118, 731-742 (2014).

${ }^{35}$ A. Ghoufi, P. Malfreyt, and D. J. Tildesley, "Computer modelling of the surface tension of the gas-liquid and liquid-liquid interface," Chem. Soc. Rev. 45, 1387-1409 (2016).

${ }^{36}$ H. D. d'Oliveira, X. Davoy, E. Arche, P. Malfreyt, and A. Ghoufi, "Test-area surface tension calculation of the graphene-methane interface: Fluctuations and commensurability," J. Chem. Phys 146, 214112 (2017).

${ }^{37}$ T. Dreher, C. Lemarchand, L. Soulard, E. Bourasseau, P. Malfreyt, and N. Pineau, "Calculation of a solid/liquid surface tension: A methodological study," J. Chem. Phys. 148, 034702 (2018).

${ }^{38}$ A. Akaishi, T. Yonemaru, and J. Nakamura, "Formation of water layers on graphene surfaces," ACS Omega 2, 2184-2190 (2017).

${ }^{39}$ A. Serva, S. Pezzotti, S. Bougueroua, D. R. Galimberti, and M.-P. Gaigeot, "Combining ab-initio and classical molecular dynamics simulations to unravel the structure of the $2 \mathrm{~d}-\mathrm{hb}$-network at the air-water interface," J. Mol. Struct. 1165, 71-78 (2018).

${ }^{40}$ M. Gaigeot and M. Sulpizi, Structure and Dynamics of Solid/Liquid Interfaces : Liquid and Biological Interfaces, edited by K. Wandelt, Vol. 7 (Wiley-VCH Verlag GmbH \& Co, 2020).

${ }^{41}$ S. Pezzotti, D. R. Galimberti, and M.-P. Gaigeot, "2d h-bond network as the topmost skin to the air-water interface," J. Phys. Chem. Lett. 8, 3133-3141 (2017).

${ }^{42}$ P. B. Petersen and R. J. Saykally, "On the nature of ions at the liquid water surface," Annu. Rev. Phys. Chem. 57, 333-364 (2006).

${ }^{43}$ D. L. McCaffrey, S. C. Nguyen, S. J. Cox, H. Weller, A. P Alivisatos, P. L. Geissler, and R. J. Saykally, "Mechanism 
of ion adsorption to aqueous interfaces: Graphene/water vs. air/water," Proc. Natl. Acad. Sci. 114, 13369-13373 (2017).

${ }^{44}$ H. Mizuno, A. M. Rizzuto, and R. J. Saykally, "Charge-transferto-solvent spectrum of thiocyanate at the air/water interface measured by broadband deep ultraviolet electronic sum frequency generation spectroscopy," J. Phys. Chem. Lett. 9, 47534757 (2018).

${ }^{45}$ E. A. Raymond, T. L. Tarbuck, M. G. Brown, and G. L. Richmond, "Hydrogen-bonding interactions at the vapor/water interface investigated by vibrational sum-frequency spectroscopy of hod/h2o/d2o mixtures and molecular dynamics simulations," J. Phys. Chem. B 107, 546-556 (2003).

${ }^{46}$ D. S. Walker and G. L. Richmond, "Understanding the effects of hydrogen bonding at the vapor-water interface: Vibrational sum frequency spectroscopy of $\mathrm{h} 2 \mathrm{o} / \mathrm{hod} / \mathrm{d} 2 \mathrm{o}$ mixtures studied using molecular dynamics simulations," J. Phys. Chem. C 111, 83218330 (2007)

${ }^{47}$ A. J. Hopkins, C. L. McFearin, and G. L. Richmond, "Investigations of the solid-aqueous interface with vibrational sumfrequency spectroscopy," Curr. Opin. Solid State Mater. Sci. 9, 19-27 (2005).

${ }^{48}$ S. Schrödle, F. G. Moore, and G. L. Richmond, "Surface speciation at solid/liquid interfaces: A vibrational sum-frequency study of acetate adsorption at the fluorite/water interface," J. Phys. Chem. C 111, 10088-10094 (2007)

${ }^{49}$ W. T. S. Cole and R. J. Saykally, "Hydrogen bond network rearrangement dynamics in water clusters: Effects of intermolecular vibrational excitation on tunneling rates," J. Chem. Phys 147 , 064301 (2017)

${ }^{50}$ W. T. S. Cole, R. S. Fellers, M. R. Viant, and R. J. Saykally, "Hydrogen bond breaking dynamics in the water pentamer: Terahertz vrt spectroscopy of a $20 \mu \mathrm{m}$ libration," J. Chem. Phys 146, 014306 (2017)

${ }^{51} \mathrm{H}$. Wang, C. Junghans, and K. Kremer, "Comparative atomistic and coarse-grained stiudy of water: what do we lose by coarsegraining ?" Eur. Phys. J. E 28, 221-229 (2009).

${ }^{52}$ G. Vasquez, E. Alvarez, and J. M. Navaza, "Surface tension of alcohol + water from 20 to $50^{\circ} \mathrm{C}$," J. Chem. Eng. Data 40,611614 (1995).

${ }^{53}$ S. Azizian and M. Hemmati, "Surface tension of binary mixtures of ethanol + ethylene glycol from 20 to $50{ }^{\circ} \mathrm{c}$," J. Chem. eng. Data 48, 662-663 (2003)

${ }^{54}$ M. A. Postigo, J. Zurita, M. L. G. A. de Soria, and M. Katz, "Excess thermodynamic properties of n-pentane + dichloromethane system at 298.15 k," Can. J. Chem. 64, 1966-1968 (2011).

${ }^{55}$ A. Zdziennicka, J. Krawczyk, K. Szymczyk, and B. Janczuk, "Components and parameters of liquids and some polymers surface tension at different temperature," Colloids and Surfaces A 529, 864-875 (2017)

${ }^{56}$ A. Erfani, S. Khosharay, and C. P. Aichele, "Surface tension and interfacial compositions of binary glycerol/alcohol mixtures," J Chem. Thermodynamics 135, 241-251 (2019).

${ }^{57}$ F. Biscay, A. Ghoufi, and P. Malfreyt, "Surface tension of wateralcohol mixtures from monte carlo simulations," J. Chem. Phys. 134, 044709 (2011)

${ }^{58}$ F. Biscay, A. Ghoufi, V. Lachet, and P. Malfreyt, "Prediction of the surface tension of the liquid-vapor interface of alcohols from monte carlo simulations," J. Phys. Chem. C 115, 86708683 (2011).

${ }^{59}$ G. Lamoureux, A. D. MacKerell, and B. Roux, "A simple polarizable model of water based on classical drude oscillators," J. Chem. Phys. 119, 5185-5197 (2003)

${ }^{60}$ G. Lamoureux, E. Harder, I. V. Vorobyov, B. Roux, and J. A. D. MacKerell, "A polarizable model of water for molecular dynamics simulationsof biomolecules," Chem. Phys. Lett. 418, 245-249 (2006)

${ }^{61}$ J. C. Neyt, A. Wender, V. Lachet, A. Ghouf, and P. Malfreyt, "Prediction of the concentration dependence of the surface tension and density of salt solutions: atomistic simulations using drude oscillator polarizable and nonpolarizable models," Phys.
Chem. Chem. Phys 15, 11679-11690 (2013).

${ }^{62}$ D. Case, R. Betz, D. Cerutti, T. C. III, T. Darden, R. Duke, T. Giese, H. Gohlke, A. Goetz, N. Homeyer, S. Izadi, P. Janowski, J. Kaus, A. Kovalenko, T. Lee, S. LeGrand, P. Li, C. Lin, T. Luchko, R. Luo, B. Madej, D. Mermelstein, K. Merz, G. Monard, H. Nguyen, H. Nguyen, I. Omelyan, A. Onufriev, D. Roe, A. Roitberg, C. Sagui, C. Simmeling, W. Botello-Smith, J. Swails, R. Walker, J. Wang, R. Wolf, X. Wu, L. Xiao, and P. Kollman, "Amber 2016," (University of California, San Francisco, 2016).

${ }^{63}$ C. J. Fennell and J. D. Gezelter, "Is the ewald summation still necessary? pairwise alternatives to the accepted standard for long-range electrostatics," J. Chem. Phys 124, 234104 (2006).

${ }^{64}$ J. M. Míguez, M. M. Piñeiro, and F. J. Blas, "Influence of the long-range corrections on the interfacial properties of molecular models using Monte Carlo simulation," J. Chem. Phys. 138 34707-34716 (2013).

${ }^{65}$ D. Cohen-Tanugi and J. C. Grossman, "Water desalination across nanoporous graphene," Nano Lett. 12, 3602-3608 (2012).

${ }^{66}$ J. L. F. Abascal and C. Vega, "A general purpose model for the condensed phases of water: TIP4P/2005," J. Chem Phys. 123 234505 (2005).

${ }^{67}$ P. Honenberg and W. Kohn, "Inhomogeneous electron gas," Phys. Rev. A 136, 864-871 (1964).

${ }^{68}$ W. Kohn and L. Sham, "Self-consistent equations including exchange and correlation effects," J. Phys. Rev. A 140, 1133-1138 (1965).

${ }^{69}$ A. D. Becke, "Density-functional exchange-energy approximation with correct asymptotic behavior." Phys. Rev. A 38, 3098 (1988).

${ }^{70}$ A. D. Becke, "Density?functional thermochemistry. iii. the role of exact exchange," J. Chem. Phys. 98, 5648-5652 (1993).

${ }^{71}$ J. P. Perdew and Y. Wang, "Accurate and simple analytic representation of the electron-gas correlation energy," Phys. Rev. E 45, 13244 (1991)

${ }^{72}$ M. J. Frisch, G. W. Trucks, H. B. Schlegel, G. E. Scuseria, M. A Robb, J. R. Cheeseman, G. Scalmani, V. Barone, G. A. Petersson, H. Nakatsuji, X. Li, M. Caricato, A. V. Marenich, J. Bloino, B. G. Janesko, R. Gomperts, B. Mennucci, H. P. Hratchian, J. V. Ortiz, A. F. Izmaylov, J. L. Sonnenberg, D. WilliamsYoung, F. Ding, F. Lipparini, F. Egidi, J. Goings, B. Peng, A. Petrone, T. Henderson, D. Ranasinghe, V. G. Zakrzewski, J. Gao, N. Rega, G. Zheng, W. Liang, M. Hada, M. Ehara, K. Toyota, R. Fukuda, J. Hasegawa, M. Ishida, T. Nakajima, Y. Honda, O. Kitao, H. Nakai, T. Vreven, K. Throssell, J. A. M. Jr., J. E. Peralta, F. Ogliaro, M. J. Bearpark, J. J. Heyd, E. N. Brothers, K. N. Kudin, V. N. Staroverov, T. A. Keith, R. Kobayashi, J. Normand, K. Raghavachari, A. P. Rendell, J. C. Burant, S. S. Iyengar, J. Tomasi, M. Cossi, J. M. Millam, M. Klene, C. Adamo, R. Cammi, J. W. Ochterski, R. L. Martin, K. Morokuma, O. Farkas, J. B. Foresman, and D. J Fox, "Gaussian 16, revision b.01," Gaussian, Inc., Wallingford CT (2016).

${ }^{73}$ C. P. Breneman and K. B. Wiberg, "Determining atom?centered monopoles from molecular electrostatic potentials. the need for high sampling density in formamide conformational analysis," J. Comput. Chem. 11, 361-373 (1990).

${ }^{74}$ I. Todorov and W. S. and, "DLPOLY3: New dimensions in molecular dynamics simulations via massive parallelism," J. Mater. Chem. 16, 1911-1918 (2006).

${ }^{75}$ M. P. Allen and D. J. Tildesley, Computer Simulation of Liquids, Second edition (Oxford: Clarendon Press, 2017).

${ }^{76} \mathrm{~S}$. Melchionna, G. Ciccotti, and B. L. Holian, "Hoover NPT dynamics for systems varying in shape and size," Mol. Phys. 78, 533-544 (1993)

77 J. H. Irving and J. Kirkwood, "The Statistical Mechanical Theory of Transport Processes .IV. The Equations of Hydrodynamics," J. Chem. Phys. 18, 817-829 (1950)

${ }^{78}$ J. S. Rowlinson and B. Widom, Molecular Theory of Capillarity (Clarendon Press, Oxford, 1982). 
${ }^{79}$ J. P. R. B. Walton, D. J. Tildesley, J. S. Rowlinson, and J. R. Henderson, "The pressure tensor at the planar surface of a liquid," Molec. Phys. 48, 1357-1368 (1983).

${ }^{80}$ J. P. R. B. Walton, D. J. Tildesley, J. S. Rowlinson, and J. R. Henderson, "The pressure tensor at the planar surface of a liquid," Molec. Phys. 50, 1381 (1983), erratum.

${ }^{81} \mathrm{M}$. Guo and B. Lu, "Long range corrections to thermodynamic properties of inhomogeneous systems with planar interfaces," J. Chem. Phys. 106, 3688-3695 (1997).

${ }^{82}$ A. Luzar and D. Chandler, "Effect of environment on hydrogen bond dynamics in liquid water," Phys. Rev. Lett. 76, 928-931 (1996).

${ }^{83}$ A. P. Willard and D. Chandler, "Instantaneous liquid interfaces," J. Phys. Chem. B 114, 1954-1958 (2010).

${ }^{84}$ J. Kessler, H. Elgabarty, T. Spura, K. Karhan, P. Partovi-Azar, A. A. Hassanali, and T. D. Kühne, "Structure and dynamics of the instantaneous water/vapor interface revisited by pathintegral and ab initio molecular dynamics simulations," J. Phys.
Chem. B 119, 10079-10086 (2015).

${ }^{85} \mathrm{~T}$. E. Goresy and R. Böhmer, "Diluting the hydrogen bonds in viscous solutions of $n$-butanol with $n$-bromobutane: A dielectric study," J. Chem. Phys 128, 154520 (2008).

${ }^{86}$ R. Böhmer, C. Gainarua, and R. Richert, "Structure and dynamics of monohydroxy alcohols - milestones towards their microscopic understanding, 100 years after debye," Phys. Rep. $\mathbf{5 4 5}$ 125-195 (2014).

${ }^{87}$ M. L. Liriano, J. Carrasco, E. A. Lewis, C. J. Murphy, T. J. Lawton, M. D. Marcinkowski, A. J. Therrien, A. Michaelides, and E. C. H. Sykes, "The interplay of covalency, hydrogen bonding, and dispersion leads to a long range chiral network: The example of 2-butanol," J. Chem Phys. 144, 094703 (2016).

${ }^{88}$ I. Essafri and A. Ghoufi, "Microstructure of nonideal methanol binary liquid mixtures," Phys. Rev. E 99, 062607 (2019).

${ }^{89}$ A. Ghoufi, F. Goujon, V. Lachet, and P. Malfreyt, "Surface tension of water and acid gases from Monte Carlo simulations," J. Chem. Phys. 128, 154716-154731 (2008). 

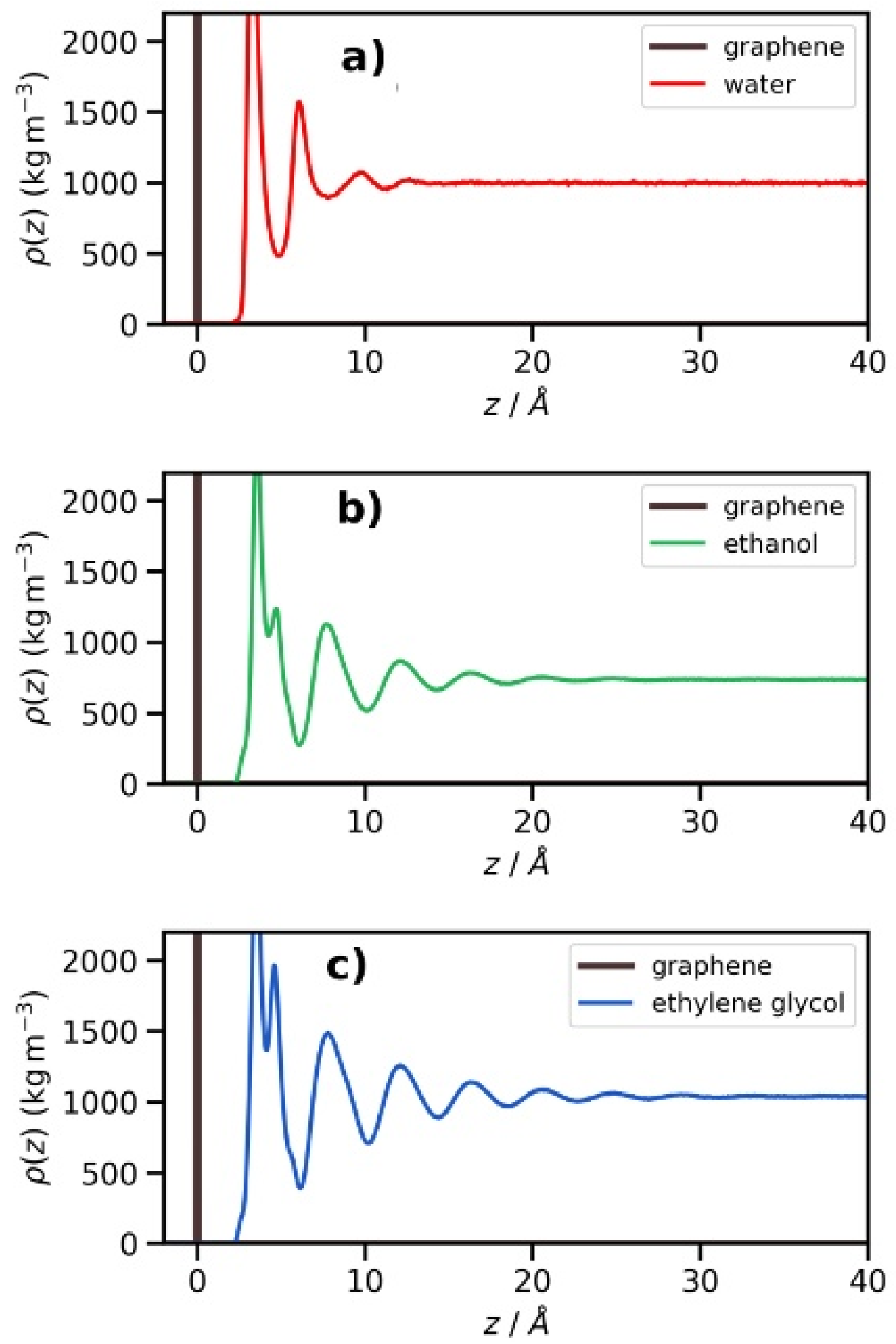


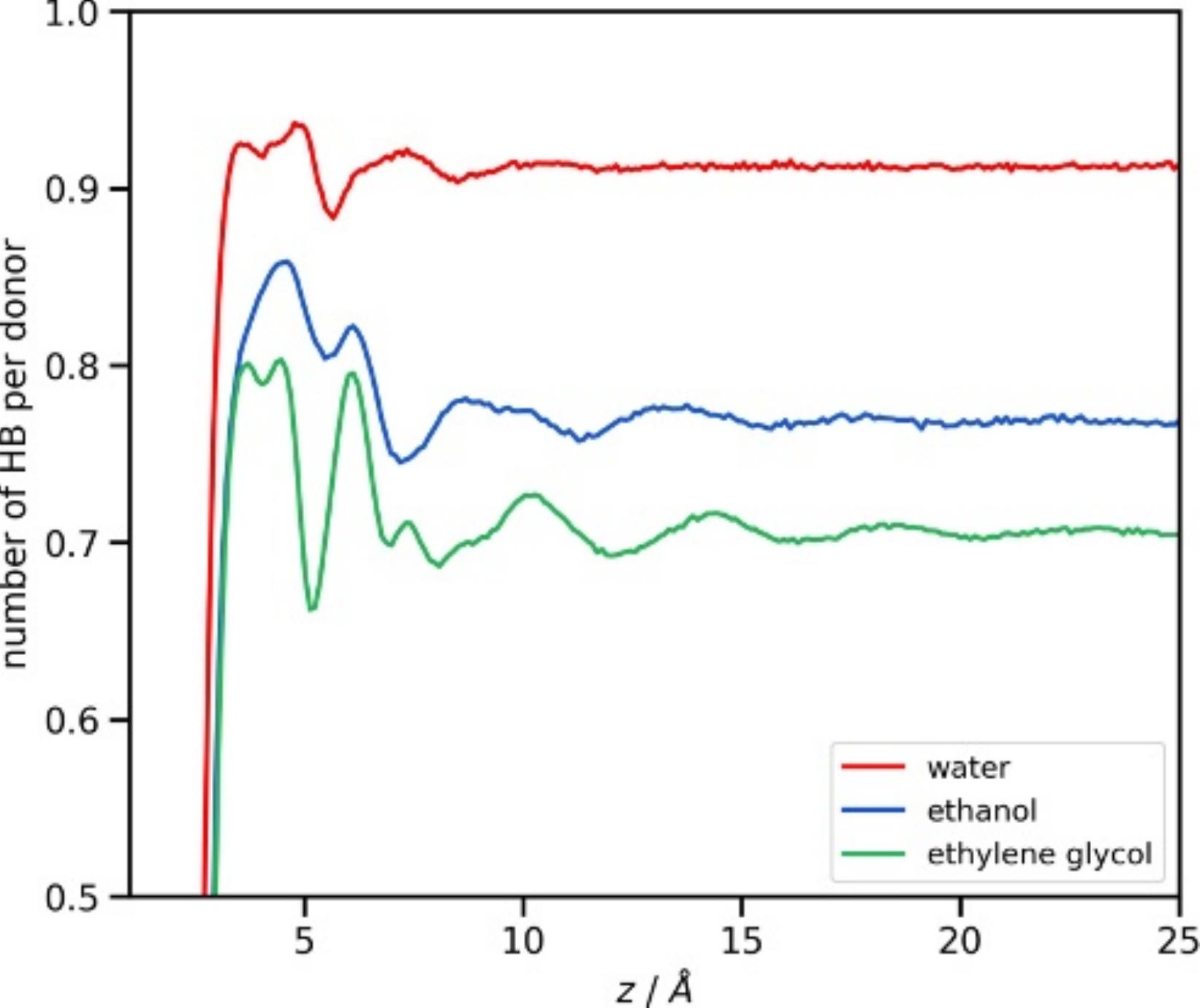




\section{WATER}

$D$
Un
$ن$

$0.5-$

$0.0-$

$-0.5-$

$-1.0$

2

4

6

8

$\begin{array}{lll}10 & 12 & 14\end{array}$

$\begin{array}{llll}16 & 18 & 20 & 22\end{array}$

ETHANOL

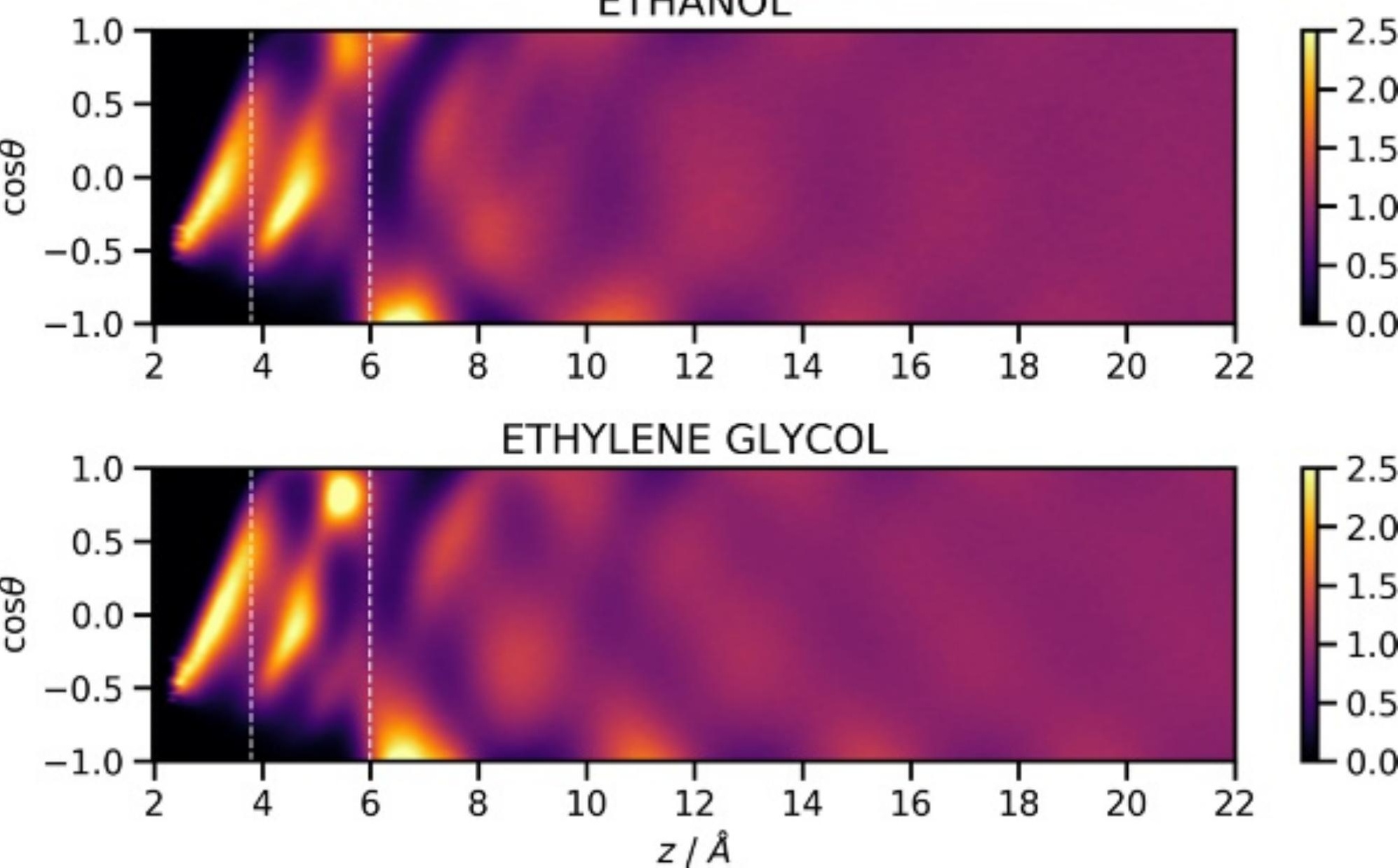

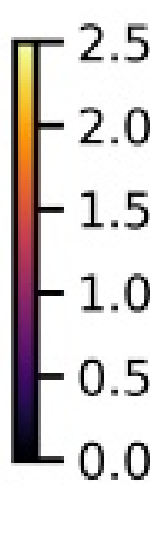
2.5 2.0 1.5 1.0 0.5 0.0

2.5
-2.0
-1.5
-1.0
-0.5
0.0 2.5 2.0 1.5 1.0 0.5 0.0 

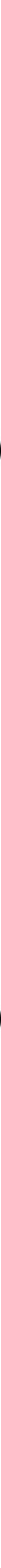

$\begin{array}{llllll}-1.00-0.75-0.50-0.25 & 0.00 & 0.25 & 0.50 & 0.75 & 1.00\end{array}$ $\cos \theta$ 

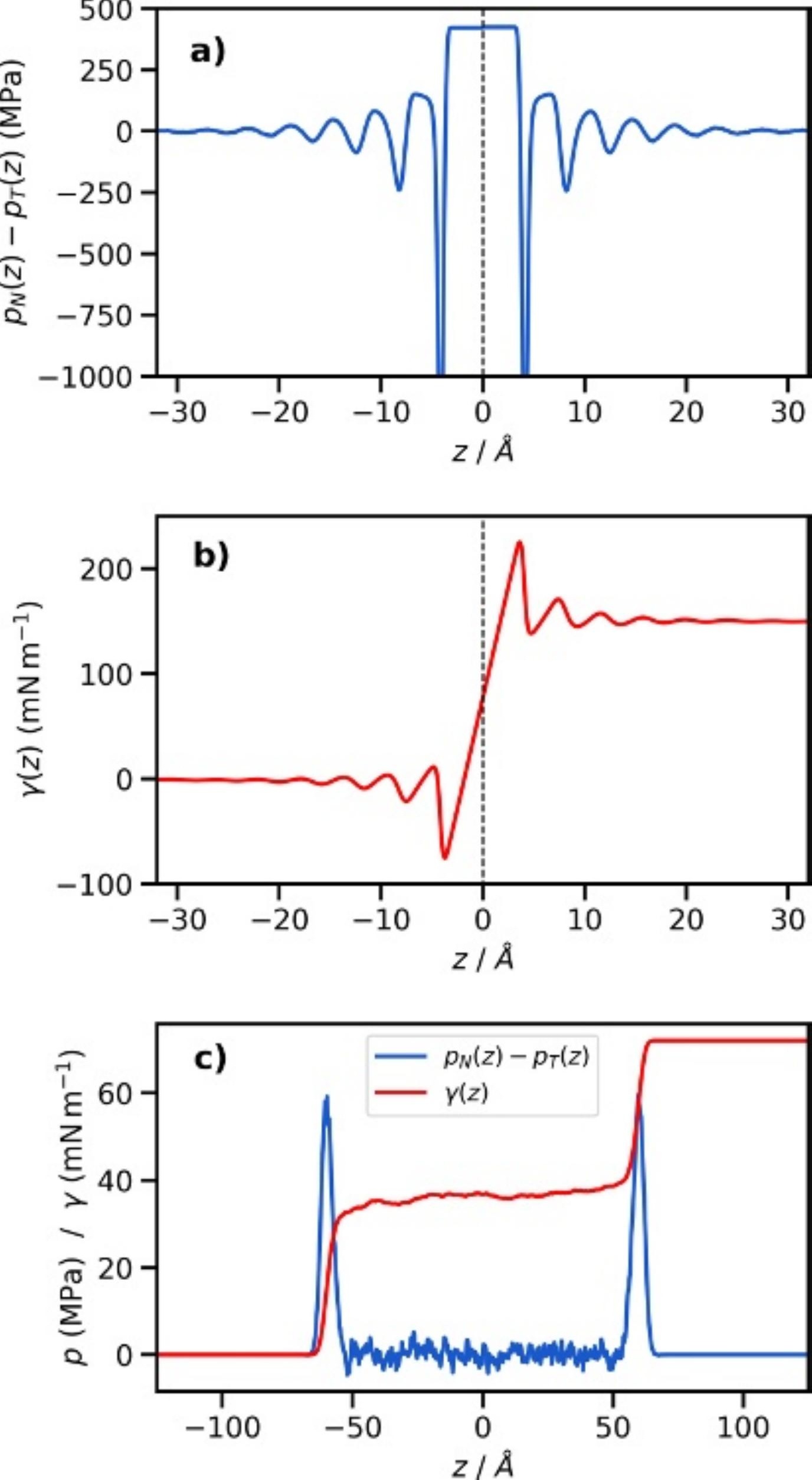
